\title{
Outcomes of Patients on Dual-Boosted PI Regimens: Experience of the Swiss HIV Cohort Study
}

\author{
Regina B. Osih,,9 Patrick Taffé, ${ }^{2}$ Martin Rickenbach, ${ }^{2}$ Angèle Gayet-Ageron, ${ }^{3}$ Luigia Elzi, ${ }^{4}$ \\ Christoph Fux, ${ }^{5}$ Milos Opravil, ${ }^{6}$ Enos Bernasconi, ${ }^{7}$ Patrick Schmid, ${ }^{8}$ Huldrych F Günthard, ${ }^{6}$ \\ Matthias Cavassini, and the Swiss HIV Cohort Study
}

\begin{abstract}
Dual-boosted protease inhibitors (DBPI) are an option for salvage therapy for HIV-1 resistant patients. Patients receiving a DBPI in the Swiss HIV Cohort Study between January1996 and March 2007 were studied. Outcomes of interest were viral suppression at 24 weeks. 295 patients (72.5\%) were on DBPI for over 6 months. The median duration was 2.2 years. Of 287 patients who had HIV-RNA $>400$ copies $/ \mathrm{ml}$ at the start of the regimen, 184 (64.1\%) were ever suppressed while on DBPI and 156 (54.4\%) were suppressed within 24 weeks. The median time to suppression was 101 days (95\% confidence interval 90-125 days). The median number of past regimens was 6 (IQR, 3-8). The main reasons for discontinuing the regimen were patient's wish (48.3\%), treatment failure $(22.5 \%)$, and toxicity $(15.8 \%)$. Acquisition of HIV through intravenous drug use and the use of lopinavir in combination with saquinavir or atazanavir were associated with an increased likelihood of suppression within 6 months. Patients on DBPI are heavily treatment experienced. Viral suppression within 6 months was achieved in more than half of the patients. There may be a place for DBPI regimens in settings where more expensive alternates are not available.
\end{abstract}

\section{Introduction}

A NTIRETROVIRAL THERAPY HAS UNDERGONE MANY CHANGES over the course of the last 20 years and has resulted in decreased morbidity and mortality among HIV-infected patients. ${ }^{1}$ However, in clinical practice, factors such as poor adherence, limited potency of prior regimens, and drug toxicity have led to an increased prevalence of multiple resistance mutations in both reverse transcriptase and protease sequences. ${ }^{2}$
Treatment options after the accumulation of several protease inhibitor (PI) mutations are limited and usually require the use of newer agents such as integrase inhibitors (raltegravir [RAL], salvage PIs (tipranavir [TPV], and darunavir [DRV]), new generation non-nucleoside reverse transcriptase inhibitors (NNRTIs) such as etravirine (ETV) or entry inhibitors (fusion [T-20] or CCR5 inhibitors (maraviroc $[\mathrm{MVC}])^{3}$ Before the availability of these new drugs, many clinicians used different salvage strategies in the treatment of

\footnotetext{
${ }^{1}$ Infectious Diseases Service, Department of Medicine, Centre Hospitalier Universitaire Vaudois and University of Lausanne, Switzerland.

${ }^{2}$ Data Coordination Center for the Swiss HIV Cohort, Lausanne, Switzerland.

${ }^{3}$ Hopital Cantonal et Universitaire de Genève, Service des Maladies Infectieuses, Geneva, Switzerland.

${ }^{4}$ University of Basel, Medicine, Division of Infectious Diseases, Basel, Switzerland.

${ }^{5}$ Universitätsspital Bern, Klinik und Poliklinik für Infektiologie, Bern, Switzerland.

${ }^{6}$ University Hospital Zürich, Division of Infectious Diseases and Hospital Epidemiology, University of Zürich, Switzerland.

${ }^{7}$ Ospedale, Civico, Department of Medicine, Division of Infectious Diseases, Lugano, Switzerland.

${ }^{8}$ Kantonsspital St. Gallen, Switzerland.

${ }^{9}$ The Reproductive Health and HIV Research Unit, University of the Witwatersrand, Johannesburg, South Africa.

Part of the results (poster P7.4/06) were presented at the $11^{\text {th }}$ European Aids Clinical Society (EACS) Conference in Madrid, Spain, October 24-27, 2007.

The members of the Swiss HIV Cohort Study are Battegay M, Bernasconi E, Böni J, Bucher HC, Bürgisser P, Calmy A, Cattacin S, Cavassini M, Dubs R, Egger M, Elzi L, Fischer M, Flepp M, Fontana A, Francioli P (President of the SHCS), Furrer H (Chairman of the Clinical and Laboratory Committee), Fux CA, Gorgievski M, Günthard HF (Chairman of the Scientific Board), Hirsch HH, Hirschel B, Hösli I, Kahlert C, Kaiser L, Karrer U, Kind C, Klimkait T, Ledergerber B, Martinetti G, Müller N, Nadal D, Paccaud F, Pantaleo G, Rauch A, Regenass S, Rickenbach M (Head of Data Center), Rudin C (Chairman of the Mother \& Child Substudy), Schmid P,Schultze D, Schüpbach J, Speck R, de Tejada BM, Taffé P, Telenti A, Trkola A, Vernazza P, Weber R, and Yerly S.
} 
multidrug-resistant HIV. Some of these regimens included two PI in addition to low-dose ritonavir (RTV, dual-boosting). RTV, a potent inhibitor of the cytochrome P450 enzymatic system, is extensively used as an adjunct to PI therapy (as a booster). ${ }^{4}$ The increase in levels of co-administered PI due to this drug-drug interaction has allowed for simpler and less toxic regimens to be adopted for the treatment of HIV. ${ }^{5}$ In addition, the enhanced pharmacokinetic profile (in particular, Cmin and Area Under the Curve, AUC) of the boosted drug enables better viral suppression ${ }^{6}$ and a higher threshold for the development of resistance than if used without RTV. ${ }^{7-9}$ Despite early studies showing marginally increased side-effects, ${ }^{5}$ boosted PI therapy has become part of the standard of care for the treatment of naïve and experienced patients, ${ }^{10,11}$ particularly because of their high genetic barrier to resistance. ${ }^{12-14}$

Similarly, the use of dual-boosted regimens has gained favor due to some studies reporting on synergistic and additive effects of PI combinations with little additive toxicity. ${ }^{15-21}$ In addition, the concept of maintaining high plasma levels of two drugs, with distinct resistance profiles, will enable each drug to retain activity against the susceptible viral quasi-species in the presence of multiple PI resistance mutations is appealing.

This approach has become more popular in recent years with the approval of atazanavir (ATV), which has less metabolic toxicity ${ }^{22}$ and therefore is deemed safer when used in addition to conventional PIs. Several pharmacokinetic studies have also shown that double-boosted protease inhibitors (DBPI) have a relatively safe profile, especially ATV co-administered with saquinavir (SQV) or lopinavir/ritonavir (LPV-r). ${ }^{16,19,20}{ }^{23}$ Few clinical observational studies showed that ATV combined with LPV-r ${ }^{23}$ was well-tolerated and efficient in patients with extensive treatment experience.

Although former recommendations issued by the international AIDS society-USA panel stated that "there are no convincing data to support the use of a DBPI and these regimens should be avoided", ${ }^{24}$ DBPI regimens were used widely in clinical practice due to lacking alternatives in salvage therapy. Despite this assertion, there are very few published studies regarding outcomes in large cohorts, and a recent small randomized controlled trial favored DBPI in an astreated analysis. ${ }^{25}$ There will likely never be a large trial to determine the relative efficacy and toxicity of the multitudes of combinations of DBPI available which could refute or confirm this hypothesis. Nevertheless, one needs to consider that in many parts of the world, widespread roll-out of antiretrovirals with low frequency of viral load monitoring is leading to the emergence of severe drug resistance. In addition, potent anti-retrovirals such as integrase inhibitors and new generation NNRTIs are unfortunately not yet available in most resource-constrained settings. ${ }^{26-28}$ This means that DBPI regimens may be the only salvage regimens available in patients who have multi-class failure in some countries. We therefore aimed to characterize the patients who have received DBPI regimens within the Swiss HIV Cohort Study (SHCS) and to identify independent factors predicting viral suppression at 6 months on a DBPI regimen.

\section{Methods}

\section{Study design}

This is a retrospective analysis of data recorded in the context of a prospective observational cohort of all patients enrolled in the SHCS since its inception (1987) and who received a treatment regimen containing RTV and two other PIs between January 1996 and March 30, 2007.

The SHCS is a longitudinal cohort that collects information on a bi-annual basis on more than 14,000 patients from seven participating centers in Switzerland. The detailed structure of the SHCS has been described elsewhere. ${ }^{29,30}$ We collected data on demographical information and results of laboratory tests at cohort visits (CD4 cell count, HIV-1 RNA, and lipid profiles), treatment regimens, and reasons for switching regimens.

\section{Definitions}

- DBPI was defined as the use of RTV in combination with two other PIs. Patients who received nelfinavir (NFV) in this combination were also included in the study, despite evidence that NFV is not significantly boosted by RTV. Patients who received newer PIs in salvage therapy, notably DRV and TPV, were excluded from the study. We also did not consider full-dose RTV in combination with SQV as a DBPI equivalent and therefore excluded patients on this particular combination from the study.

- HIV RNA suppression was defined as a viral load of $<400$ copies $/ \mathrm{ml}$. We chose this cutoff because it most accurately identifies all episodes of suppression across the time-span chosen within the cohort. Cutoffs for suppression were changed during the 10 years of observation that we chose (from $<400$ copies $/ \mathrm{ml}$ to $<20$ copies $/ \mathrm{ml}$ ) with the final cutoff recorded in the cohort being dependent on the laboratory method used at a given time. Additionally, patients who were considered suppressed were assigned an RNA value of zero in the database and therefore the real value for these patients cannot be determined retrospectively.

\section{Laboratory values}

CD4 count and HIV RNA viral load at cohort entry were defined as the first value recorded within the cohort. These values do not necessarily represent the laboratory values present at the time of HIV-1 diagnosis for each patient.

CD4 count and HIV RNA viral load at the time of DBPI start were calculated within a time range so as to most accurately reflect the values available to the clinician. For CD4 counts, we considered all values within 30 days of the event of interest (cohort start, DBPI start, DBPI stop, cohort exit) and chose the value closest to the date of the event within those 30 days. Since HIV RNA is more often used for clinical decision making, we took into consideration values that were closest to the sixth month after DBPI start, up to 30 days before but no more than 10 days after. The value of HIV-RNA up to 10 days after the cohort visit would be an accurate reflection of the value available to the clinician at the time clinical decisionmaking occurred.

For HDL and cholesterol values, we chose the value that was closest to the event of interest, either before or after the event but within 30 days.

\section{Treatment interruptions and changes}

Any instance where the patient recorded as not being on therapy either before or after the regimen of interest was 
considered as a treatment interruption, regardless of the duration or reason for the treatment interruption (intercurrent illness, physician's decision or patient's decision for example).

The number of regimen changes was calculated based on the change of any drug within the regimen, and was not limited to the PI class.

The duration of zidovudine (AZT) monotherapy and nucleoside reverse transcriptase inhibitors (NRTI) bi-therapy alone is based on the cumulative time the patient received these regimens, irrespective of treatment interruptions within this time. However, patients had to have gone back to the original regimen in order for the time to be included (i.e., patients who switched from AZT to bi-therapy and then back to AZT were not considered to be on AZT monotherapy for that entire time period). NNRTI experience was defined as the receipt of a regimen containing efavirenz (EFV), nevirapine (NVP), or delavirdine (DLV) before the onset of the DBPI regimen, regardless of the concomitant drugs in the regimen.

NNRTI co-administration was defined as the receipt of EFV or NVP in conjunction with the DBPI regimen.

\section{Outcomes}

Outcomes of interest were characteristics of the patients who received DBPI, particularly for those who received the regimen for less than 6 months versus those who continued the regimen for longer periods, time to viral suppression for all patients included in the study, and the proportion of patients who achieved a viral suppression in less than 180 days after the start of the DBPI regimen. In addition, factors associated with duration of DBPI therapy were investigated.

\section{Statistical analysis}

Simple descriptive statistics were used to describe the study population. To compare the group that achieved suppression to the one that did not, the Pearson chi-square test was used for categorical data, as well as Fisher's exact test when required, and Student $t$-test, as well as Wilcoxon rank sum test for continuous data. The logistic regression model was used for dichotomous outcomes. Adjustment was performed for at most one or two factors at a time to assess conditional associations. No specific model selection was performed and results are mainly descriptive.

\section{Results}

\section{Baseline characteristics}

We identified 407 patients who received DBPI during the study period and 295 patients who received DBPI for at least 6 months. Patients who received more than 6 months of treatment and those who stopped early did not differ significantly in age, gender distribution, mode of HIV acquisition, regimens used, or cohort outcome. However, patients who received DBPI for longer than 6 months tended to be different from those who stopped the treatment earlier regarding HIV RNA at DBPI start (4.6 log vs. $3.6 \log , p<0.01)$, mean CD4 counts at enrollment (260 vs. 356.5, $p=0.02$ [not importantly significant]), and a tendency for lower CD4 count at DBPI start (187 vs. $225, p=0.05$, idem). Moreover, more patients were experiencing virological failure at the time of DBPI start in the group who pursued treatment for more than 6 months $(73.6 \%$ vs. $61.6 \%, p=0.02)$. The two groups also differed with respect to treatment experience before DBPI start. Patients who stopped early ( $<6$ months) had been on antiretroviral treatment for a median of 5.9 years (interquartile range [IQR] 3.1-8.8) compared to 7.3 years (IQR 4.9-9.2) in patients who continued DBPI for longer $(p<0.01)$.

\section{Characterization of DBPI group}

We will then describe the 295 patients who underwent DBPI treatment for at least 6 months. They had a median age of 43 years (IQR: 38-49) and were mostly male (76.9\%, $n=227)$. The most common risk factor for HIV acquisition was men-having-sex-with-men (MSM) $(43.4 \%, n=128)$, followed by heterosexual contact $(29.8 \%, n=88)$, and intravenous drug use $(23.1 \%, n=68)$. Eleven patients had either an unknown risk factor or acquired HIV through blood transfusions. A total of 248 patients $(84.1 \%)$ were still active in the cohort at the time of the last follow-up visit. Thirty-three patients died $(11.2 \%)$ since they were first enrolled between January 1996 and March 30, 2007 and only 14 (4.7\%) had either withdrawn from the SHCS or were lost to follow-up on March 30, 2007. The cause of death was related to HIV in 17 patients (5.8\%). Table 1 summarizes the characteristics of the patients as well as the treatment experience before starting therapy.

\section{Treatment interruptions}

To better characterize the treatment experience of our cohort, we analyzed the number of treatment interruptions that had occurred before the start of DBPI as well as the number of regimen changes before and after the DBPI regimen. Ninety patients $(30.5 \%)$ had never interrupted their treatment before starting the DBPI regimen, 102 patients (34.6\%) had interrupted a regimen once, while $94(31.9 \%)$ had done so two to five times. There were 9 patients $(3.1 \%)$ who had between six and ten treatment interruptions prior to starting DBPI. For the regimen changes, 135 patients $(45.8 \%)$ experienced between one and five regimen changes prior to starting DBPI. 116 (39\%) had 6-10 changes and 33 (11.2\%) had 11-15 changes. There were 9 patients $(3.1 \%)$ who had more than 15 different regimens from the time of enrollment. The median number of changes after the DBPI regimen was 2 (IQR:1-4) (Table 1).

\section{$D B P I$ regimens used and duration on therapy}

LPV-r was the most common PI used in our DBPI cohort. A total of $240(81.4 \%)$ patients were receiving LPV-r in combination with one other PI. The most common combination was LPV-r/amprenavir (LPV-r/APV) used in 110 (37.3\%) patients, followed by LPV-r/SQV in $82(27.8 \%)$ patients. SQV-r/ ATV was given to 36 (12.2\%) patients. Eighty-eight $(29.8 \%)$ patients also received concomitant NNRTI and 19 (6.4\%) were receiving fusion inhibitors. The number of drugs in the regimen ranged from three to more than six, with 129 patients receiving four drugs $(43.7 \%)$ and $15(5.1 \%)$ receiving more than six drugs concomitantly. We subdivided the cohort according to the year DBPI therapy was begun and found that $227(76.9 \%)$ patients had started DBPI after the year 2000 and only 68 patients had been started on a DBPI regimen before the year 2000 (Table 2).

The DBPI regimen was stopped in 120 (40.7\%) patients before the end of the follow-up period. The most common 
Table 1. Baseline Characteristics and Treatment Experience of Patients on Dual-Boosted Protease Inhibitors in the Swiss HIV Cohort Study, January 1996-March 2007

\begin{tabular}{|c|c|c|}
\hline Variable (unit) & $\begin{array}{c}\text { All patients } \\
N=407(\%),[I Q R]\end{array}$ & $\begin{array}{c}D B P I>6 \text { months } \\
N=295(\%),[I Q R]\end{array}$ \\
\hline Time from ART start to DBPI start (years) & $7.0(4.4-9.1)$ & $7.3(4.9-9.2)$ \\
\hline \multicolumn{3}{|l|}{ Median time on DBPI } \\
\hline Days & $520(159-1126)$ & $799(421-1443)$ \\
\hline Years & $1.4(0.4-3.1)$ & $2.2(1.2-3.9)$ \\
\hline CD4 Nadir (median, cells $/ \mathrm{mm}^{3}$ ) & $66(20-151)$ & $61(16-136)$ \\
\hline D4 at start of cohort (median, cells $/ \mathrm{mm}^{3}$ ) & $286(134-470)$ & $260(120-445)$ \\
\hline CD4 at last follow-up or exit (median, cells $/ \mathrm{mm}^{3}$ ) & $360(163-533)$ & $361(183-539)$ \\
\hline RNA at start of cohort $\left(\log _{10}\right)$ & $4.6(3.5-5.2)$ & $4.7(3.7-5.2) n=240$ \\
\hline RNA at start of DBPI treatment $\left(\log _{10}\right)$ & & $4.6 \log (3.4-5.2]$ \\
\hline Azt monotherapy exposure & $198(48.9)$ & $153(52)$ \\
\hline NRTI bi-therapy & $265(65.4)$ & $201(68.1)$ \\
\hline Single PI exposure before starting DBPI & $332(81.9)$ & $251(85.4)$ \\
\hline NNRTI exposure before starting DBPI & $250(61.7)$ & $181(61.6)$ \\
\hline RTV-SQV experience before DBPI start & $179(44)$ & $134(45.4)$ \\
\hline $\begin{array}{l}\text { Number of treatment interruptions } \\
\text { before DBPI start (median) }\end{array}$ & $1[0-2]$ & $1[0-2]$ \\
\hline None & $116(28.5)$ & $90(30.5)$ \\
\hline One & $137(47.1)$ & $102(34.6)$ \\
\hline Two-Five & $142(48.8)$ & $94(31.9)$ \\
\hline Six-Ten & $12(4.1)$ & $9(3.1)$ \\
\hline Number of regimen changes before DBPI start & 6 [3-9] & $6[3-8]$ \\
\hline None & $24(9.4)$ & $15(5.1)$ \\
\hline $1-5$ & $172(42.3)$ & $122(41.4)$ \\
\hline $6-10$ & $152(37.3)$ & $116(39.0)$ \\
\hline $11-15$ & $49(12.0)$ & $33(11.2)$ \\
\hline$>16$ & $10(2.5)$ & $9(3.1)$ \\
\hline Number of Changes after DBPI stop & $2[1-4]$ & $2[1-4]$ \\
\hline \multicolumn{3}{|l|}{ DBPI Regimen } \\
\hline Containing LPV/r & $318(78.1)$ & $240(81.4)$ \\
\hline Containing SQV & $174(42.7)$ & $130(44.1)$ \\
\hline Containing RTV & $104(25.5)$ & $58(19.7)$ \\
\hline Containing APV & $141(34.6)$ & $116(39.4)$ \\
\hline Containing ATV & $96(23.6)$ & $63(21.4)$ \\
\hline Containing IDV & $41(10.1)$ & $17(5.8)$ \\
\hline Containing fos-APV & $14(3.4)$ & $13(4.4)$ \\
\hline Concomitant NNRTI & $111(27.3)$ & $88(29.8)$ \\
\hline Concomitant T-20 & $25(6.1)$ & $19(6.4)$ \\
\hline \multicolumn{3}{|l|}{ Number of drugs in regimen } \\
\hline 2 & $16(3.9)$ & $13(4.4)$ \\
\hline 3 & $101(24.8)$ & $84(28.5)$ \\
\hline 4 & $179(44.0)$ & $129(43.7)$ \\
\hline 5 & $84(20.6)$ & $54(18.31)$ \\
\hline$\geq 6$ & $27(6.6)$ & $15(5.1)$ \\
\hline
\end{tabular}

reason for stopping was provider decision or patient preference $(48.3 \%, n=58)$. Treatment failure accounted for the withdrawal of the regimen in $27(22.5 \%)$ and toxicity in 19 $(15.8 \%)$ patients. In these 19 patients, dyslipidemia, elevated cardiovascular risk, and abnormal fat distribution prompted the regimen change in $8(42.1 \%)$ cases, and GI symptoms, including elevated liver enzymes, were present in 7 (36.9\%). The remaining 4 patients discontinued due to endocrine, nervous system, or other unspecified toxicities.

Of note when compared to the group of patients who had early switches and never received DBPI for longer than 6 months, toxicity accounted for the treatment withdrawal in 29 $(35.4 \%)$ of the 112 patients. This difference was statistically significant $(p=0.02)$. There were no significant differences in the group who stopped early compared to the group who received treatment for over 6 months with respect to patient or provider preference as a reason for stopping the regimen or the occurrence of virological failure.

\section{Virological response to $D B P I$ treatment}

Virological suppression defined as a HIV RNA < 400 copopies/ml was observed in 184 (64.1\%) of the 287 patients who had a virological failure at the start of the DBPI regimen and $156(54.4 \%)$ achieved suppression within 24 weeks of starting the regimen. Of all 287 patients, 170 (79.1\%) who are still in the cohort by the end of May 2009 were suppressed at their last follow-up appointment and $148(68.8 \%)$ had an HIV-RNA of 
Table 2. Characteristics of Patients Who Achieved Suppression in Less Than 6 months and Who Received More Than 6 Months of DBPI, and Were Failing Therapy at the Start of DBPI

\begin{tabular}{|c|c|c|c|}
\hline Variable & $\begin{array}{l}R N A<400 \text { copies } / m l \\
N=141(\%),[I Q R]\end{array}$ & $\begin{array}{c}R N A>400 \text { copies } / m l \\
N=77(\%),[I Q R]\end{array}$ & p Value \\
\hline Age & 43.9 [38-49] & $42[37-46]$ & 0.11 \\
\hline Ethnicity (Caucasian) & $120(85.1)$ & $71(92.2)$ & 0.13 \\
\hline Gender (male) & $107(75.9)$ & $63(81.2)$ & \\
\hline Riskgroup & & & 0.01 \\
\hline Heterosexual & 46 (32.6) & $21(27.7)$ & \\
\hline IDU & $41(29.1)$ & $10(12.9)$ & \\
\hline MSM & $49(34.7)$ & $42(54.6)$ & \\
\hline Other & $5(3.6)$ & $4(5.2)$ & \\
\hline Mortality & $10(7.1)$ & 17 (22.1) & 0.0013 \\
\hline CD4 at start of DBPI (median) & $184.4(73.5-256.5)$ & $162.3(21-242)$ & 0.081 \\
\hline \multicolumn{4}{|l|}{ CD4 Category at start } \\
\hline$<200$ cells $/ \mathrm{ml}$ & $73(58.9)$ & $46(65.7)$ & 0.51 \\
\hline $200-350$ cells $/ \mathrm{ml}$ & 38 (30.6) & $16(22.9)$ & \\
\hline$>350$ cells $/ \mathrm{ml}$ & $13(10.5)$ & $8(11.4)$ & \\
\hline CD4 at cohort entry (median) & $295.7(79-390)$ & $341.5(174-450)$ & 0.013 \\
\hline VL at cohort entry $\left(\log _{10}\right)$ & $4.41(3.9-5.1)$ & $4.68(4.1-5.2)$ & 0.045 \\
\hline VL at start of DBPI $\left(\log _{10}\right)$ & $4.56(3.7-5.2)$ & $4.95(4.7-5.4)$ & 0.0011 \\
\hline \multicolumn{4}{|l|}{ Treatment year (start) } \\
\hline 1996-1999 & $2(1.4)$ & $8(10.4)$ & 0.002 \\
\hline 2000-2002 & $72(51.1)$ & $48(62.3)$ & \\
\hline 2003-2004 & $44(31.2)$ & $14(18.2)$ & \\
\hline$>2004$ & $23(16.3)$ & $7(9.1)$ & \\
\hline Year of DBPI start & & & 0.0003 \\
\hline Before 2000 & $28(19.9)$ & $33(42.9)$ & \\
\hline After 2000 & $113(80.2)$ & $44(57.1)$ & \\
\hline RTV-SQV before DBPI start & $57(40.4)$ & $44(57.2)$ & 0.02 \\
\hline AZT monotherapy (received) & $76(53.9)$ & $44(57.1)$ & 0.64 \\
\hline NRTI bitherapy (received) & $95(67.4)$ & $55(71.4)$ & 0.54 \\
\hline \multicolumn{4}{|l|}{ Regimen used } \\
\hline LPV-r/AMP & $58(41.2)$ & $41(53.2)$ & 0.09 \\
\hline LPV-r/SQV & $43(30.5)$ & $11(14.3)$ & 0.008 \\
\hline LPV-r/ATV & $9(6.4)$ & $1(1.3)$ & 0.09 \\
\hline LPV-r/fos-AMP & $6(4.3)$ & $3(3.9)$ & 0.99 \\
\hline SQV/RTV & $18(12.8)$ & 15 (19.5) & 0.23 \\
\hline SQV-r/AMP & $1(0.71)$ & $2(2.6)$ & 0.28 \\
\hline Number of regimen changes & & & 0.09 \\
\hline 0 & $1(0.7)$ & 0 & \\
\hline $1-5$ & $68(48.2)$ & $34(44.2)$ & \\
\hline $6-10$ & $55(39.0)$ & $24(31.2)$ & \\
\hline$>11$ & 17 (12.1) & $19(24.7)$ & \\
\hline Number of treatment interruptions & & & 0.79 \\
\hline 0 & $38(26.9)$ & $19(24.7)$ & \\
\hline $1-2$ & 77 (54.6) & $41(53.2)$ & \\
\hline$>2$ & $26(18.4)$ & $17(22.1)$ & \\
\hline
\end{tabular}

less than 50 copies $/ \mathrm{ml}$ at their last follow-up. The median time to suppression was 101 days (95\% confidence interval, 95\% CI: 90-125 days).

We compared the baseline characteristics of the patients who achieved suppression in the first 24 weeks and those who did not in an "intention to treat analysis" (i.e., irrespective of the treatment duration). Patients who achieved suppression were more likely to be intravenous drug users $(p=0.01)$, had a lower CD4 at cohort entry $(p=0.01)$, and a lower HIV-RNA at the start of DBPI therapy, and were more likely to start DBPI after the year 2000. In terms of outcomes, patients who did not achieve suppression had a higher mortality $(22.1 \%$ vs. $7.1 \%, p=0.001)$, and had a lower median CD4 gain during therapy ( +96.6 vs. +195.2 cells $\left./ \mathrm{mm}^{3}, p<0.0001\right)$. These patients also differed significantly in terms of exposure to SQVRTV before starting DBPI and the use of LPV-r/SQV as a DBPI regimen (Table 2).

\section{Toxicity}

Regarding toxicity of DBPI, there was no significant difference in lipid values before and after treatment in the 120 patients who had a lipid profile available within 100 days of starting or stopping the DBPI regimen. The mean change in cholesterol values before and after DBPI therapy was $-0.49 \mathrm{mmol} / 1(95 \% \mathrm{CI}:-0.96$ to $-0.02 ; p=0.05)$ and the mean 
Table 3. Multiple Logistic Regression: Factors Associated with Early Suppression (<24 Weeks) in Patients who Received DBPI Therapy and Had HIV RNA > 400 Copies/ml at Start of DBPI

\begin{tabular}{|c|c|c|c|c|}
\hline \multirow[b]{2}{*}{ Variable } & \multicolumn{2}{|c|}{ In all patients $n=287$} & \multicolumn{2}{|c|}{$\begin{array}{l}\text { In patients who received therapy } \\
\text { for }>6 \text { months, } n=218\end{array}$} \\
\hline & OR $(95 \% C I)$ & p Value & OR $(95 \% C I)$ & p Value \\
\hline HIV-1 RNA at start & - & - & $0.49(0.33-0.75)$ & 0.0008 \\
\hline Treatment before 2000 & $0.10(0.023-0.46)$ & 0.003 & $0.08(0.01-0.44)$ & 0.04 \\
\hline Intravenous drug use & $2.29(1.26-4.18)$ & 0.007 & $2.32(1.04-5.14)$ & 0.04 \\
\hline Regimens used & & & & \\
\hline Lopinavir-r/Atazanavir & $3.95(1.06-14.72)$ & 0.04 & - & - \\
\hline Lopinavir-r/Saquinavir & $2.04(1.10-3.65)$ & 0.003 & $2.56(1.16-5.62)$ & 0.02 \\
\hline
\end{tabular}

change in HDL was $+0.04 \mathrm{mmol} / 1$ (95\% CI -0.08 to 0.09 ; $p=0.89)$.

\section{Factors associated with virological response}

A multivariable regression model was built to determine which factors were associated with early suppression in both patient groups. We showed that the transmission of HIV through intravenous drug use, the start of DBPI after 1999 as well as the use of LPV-r/SQV and LPV-r/ATV were all independently associated with an early suppression of HIV-1 RNA. However, in patients who received DBPI for longer than 6 months, HIV-1 RNA at treatment start was strongly associated with early suppression (OR 0.49 95\% CI 0.33-0.75, $p=0.0008$ but treatment with LPV-r/ATV was not (Table 3).

\section{Discussion}

To our knowledge, our study is the largest published to date describing the use of DBPI in extensively treatmentexperienced patients in detail. Several previous studies attempting to evaluate the effectiveness of DBPI in HIV treatment have been discontinued ${ }^{25}$ and a prospective trial is unlikely to be undertaken given the newer regimens available for therapy.

Our analysis shows that patients who received LPV-r/SQV as part of their DBPI regimen were more likely to achieve suppression, especially when the year of initiation of DBPI therapy was after 2000. This may also be related to the introduction of newer NRTIs such as tenofovir or abacavir, which may have retained some activity in patients who had thymidine analog mutations from exposure to single or dual NRTI regimens in the past. Of the patients who continued to receive DBPI, the proportion who achieve virologic suppression $(64.1 \%)$ was comparable and in some instances, better than, those described in treatment-naïve studies in the earlier years of HIV treatment. ${ }^{31}$ Our findings also suggest comparable suppression rates than in "real-life" settings with other salvage approaches studied in randomized controlled trials such as the use of newer PIs, NNRTIs, and integrase inhibitors with optimized background regimens. ${ }^{32-34}$ In addition, the concomitant use of NNRTI did not influence the outcome and both NNRTIs and enfuviritide were used by a minority of patients (20.5\% and $5.4 \%$, respectively). In this study, $64.6 \%$ of the 218 patients who started a DBPI due to virological failure and continued the treatment for longer than 6 months, achieved a viral load of $<400$ copies $/ \mathrm{ml}$. All the patients were heavily treatment experienced, with an average of six regimen changes before starting a DBPI regimen. Patients infected through intravenous drug use show better virological suppression. This may be explained by the fact that patients who are current intravenous drug users are more often directly observed and therefore may have better adherence than other patients in the cohort. While toxicity was not an issue for patients who continued on DBPI for longer than 6 months, it was a significant reason to stop early, indicating thereby that early toxicity is a barrier to wider use of DBPI (at a mean time of 41 days, [IQR 10.5-95]). In addition, the patients who stayed on DBPI for longer than 6 months had a lower CD4 count, a higher viral load and were more treatment-experienced at the start of DBPI compared to those who discontinued the treatment earlier. This may represent a treatment bias towards maintenance of DBPI regimen in patients with a more advanced disease and higher risk for progression to AIDS.

Several small series have been published and suggested that DBPI-based salvage regimens may be beneficial to patients and were responsible of few toxicity. ${ }^{35-38}$ In contrast, Petersen et al. published results from a retrospective cohort study comparing the efficacy of DBPI versus boosted single PI therapy in 183 and 805 patients, respectively, and concluded that there was no statistically significant benefit to use DBPI for salvage therapy. However, the findings did suggest that there may be a moderate size benefit if the cohort had been larger. ${ }^{39}$ The authors also point out the limitations of using a retrospective analysis of data compared to the gold standard of a randomized doubleblind clinical trial. Similarly, Loutfy et al. found that the addition of APV to a salvage regimen containing LPV $/ r$ was not associated with a faster time to achieve virological suppression nor with a difference in virological rebound rates. ${ }^{40}$ Another recent clinical trial in Thailand reported on 50 treatmentexperienced children who received SQV in combination to $\mathrm{LPV} / \mathrm{r}$ and found that there was significant rise in CD4 counts and viral suppression $<400$ and $<50$ at 48 weeks was achieved in $78 \%$ and $64 \%$ of cases, respectively. ${ }^{41}$ An earlier study by the same group found that the pharmacokinetic profile of this regimen was favorable in children. ${ }^{42}$ However, APV or SQV in combination with LPV/r have fallen out of favor in recent years due to the side-effect profile on lipids as well as due to the high pill-burden, leading to a risk of reduced adherence. In addition, results on pharmacokinetic interactions of APV and $\mathrm{LPV} / \mathrm{r}$ have produced conflicting results. Fosamprenavir, in combination with $\mathrm{LPV} / \mathrm{r}$, has also shown to have antagonistic pharmacokinetic profiles and LPV/r reduces fAPV levels. ${ }^{25}$ 
The conflicting results published so far on the general use of DBPI may also have been related to the side-effect profile and potency of the individual PIs studied, while studies using newer agents were more likely to report positive findings. ${ }^{23}$ More recently, a review of published studies on DBPI concluded that those combinations may play a positive role in settings where other drugs are not available. ${ }^{43}$

Our study presents several limitations due to its observational nature exposing to potential misclassification and selection bias. Patients were first not randomized and were not compared to a controlled group, leading to the risk of selection bias as information bias. Second, adherence data has only systematically been collected in the SHCS since $2003,{ }^{44}$ and thus was not available for this study. Third, genotypic drug resistance information was not sufficiently available for the current study because genotyping was only prospectively used widely after introduction into the SHCS in the year $2000{ }^{2}$ However, one may argue that at the time when DBPI regimens were widely instituted, genotypic drug resistance information was only sparsely available, exactly reflecting the situation as it presents today in the developing countries, where drug resistance testing will not be routinely available in the near future. These countries however are exactly the ones that might have to depend mostly on DBPI treatments for salvage in the future. In addition, the main criticism against the use of DBPI in clinical practice was its potential for higher toxicity, especially regarding the higher risk for cardiovascular morbidity. We were able to report before and after lipid profiles in only 47 patients, and even though this did not have any significant increase in cholesterol, a more in-depth study would be necessary to confirm these results. A retrospective pharmacokinetic analysis performed on stored serum samples could have yielded more information on drug levels and the exact nature of drug interactions. However, this would not be possible in the present study since information about timing of the last dose is not available and the results of any pharmacokinetic data would therefore be difficult to interpret.

In conclusion, this retrospective analysis of a large prospective observational database shows that virological suppression with DBPI was reached in $64 \%$ in a highly antiretroviral drug-exposed population. These regimens seem to be well-tolerated with less than $20 \%$ toxicity, and hyperlipidemia did not seem to occur at a statistically significant level. Moreover, $73 \%$ of patients tolerated the DBPI for a median of 2.2 years [IQR, 1.2-3.9]. Even though potentially safer alternatives are currently marketed in the treatmentexperienced patients, DBPI salvage regimens may only be the one available for most people in resource-poor settings. Non clade-B viruses are well represented in the SHCS database, ${ }^{45}$ but in our overall study population, 45 (14\%) of the subtyped viruses were non-B viruses and 1 patient was infected with HIV-2. However, there is no evidence in the literature that non-B viruses and HIV-2 would differ in their response to DBPI. Therefore this salvage strategy deserves further consideration in resource-poor settings as resistance to first-line regimens, and particularly to the entire class of NRTI and first generation NNRTIs, seems to develop at a high rate due to the absence of viral load monitoring. ${ }^{26-28}$ Therefore, just as heavily experienced patients in the SHCS had a benefit from DBPI regimens at time when other therapies were not available, these regimens may also represent a bridge of survival before more expensive drugs, widespread viral load testing, and genotypes are widely made available for patients in resource-limited settings who fail current treatments.

\section{Author Disclosure Statement}

This study has been financed in the framework of the Swiss HIV Cohort Study (project $\mathrm{n}^{\circ} 525$ ), supported by the Swiss National Science Foundation (SNF Grant \#3345-062041)

\section{References}

1. Bartlett JA, Fath MJ, Demasi R, et al.: An updated systematic overview of triple combination therapy in antiretroviralnaive HIV-infected adults. AIDS 2006;20:2051-2064.

2. von Wyl V, Yerly S, Burgisser $P$, et al.: Long-term trends of HIV type 1 drug resistance prevalence among antiretroviral treatment-experienced patients in Switzerland. Clin Infect Dis 2009;48:979-987.

3. Youle M, Staszweski S, Clotet B, et al.: Concomitant use of an active boosted protease inhibitor with enfuvirtide in treatment-experienced, HIV-infected individuals: Recent data and consensus recommendations. HIV Clin Trials 2006;7:86-96.

4. Gallant JE. Protease-inhibitor boosting in the treatmentexperienced patient. AIDS Rev 2004;6:226-233.

5. Arnaiz JA, Mallolas J, Podzamczer D, et al.: Continued indinavir versus switching to indinavir/ritonavir in HIV-infected patients with suppressed viral load. AIDS 2003;17:831-840.

6. Boffito M, Maitland D, Samarasinghe Y, and Pozniak A. The pharmacokinetics of HIV protease inhibitor combinations. Curr Opin Infect Dis 2005;18:1-7.

7. Kempf DJ, King MS, Bernstein B, et al.: Incidence of resistance in a double-blind study comparing lopinavir/ritonavir plus stavudine and lamivudine to nelfinavir plus stavudine and lamivudine. J Infect Dis 2004;189:51-60.

8. Voigt E, Wasmuth JC, Vogel M, et al.: Safety, efficacy and development of resistance under the new protease inhibitor lopinavir/ritonavir: 48-week results. Infection 2004;32:82-88.

9. Hirsch MS, Gunthard HF, Schapiro JM, et al.: Antiretroviral drug resistance testing in adult HIV-1 infection: 2008 recommendations of an International AIDS Society-USA panel. Clin Infect Dis J 2008;47:266-285.

10. Hammer SM, Vaida F, Bennett KK, et al.: Dual vs single protease inhibitor therapy following antiretroviral treatment failure: A randomized trial. JAMA 2002;288:169-180.

11. Hammer SM, Eron JJ, Jr., Reiss P, et al.: Antiretroviral treatment of adult HIV infection: 2008 recommendations of the International AIDS Society-USA panel. JAMA 2008;300:555-570.

12. Riddler SA, Haubrich R, DiRienzo AG, et al.: Class-sparing regimens for initial treatment of HIV-1 infection. N Engl J Med 2008;358:2095-2106.

13. von Wyl V, Yerly S, Boni J, et al.: Factors associated with the emergence of K65R in patients with HIV-1 infection treated with combination antiretroviral therapy containing tenofovir. Clin Infect Dis 2008;46:1299-1309.

14. von Wyl V, Yerly S, Boni J, et al.: Emergence of HIV-1 drug resistance in previously untreated patients initiating combination antiretroviral treatment: A comparison of different regimen types. Arch Intern Med 2007;167:1782-1790.

15. Boffito M, Dickinson L, Hill A, et al.: Steady-state pharmacokinetics of saquinavir hard-gel/ritonavir/fosamprenavir in HIV-1-infected patients. J Acquir Immune Defic Syndr 2004;37:1376-1384.

16. Boffito M, Kurowski M, Kruse G, et al.: Atazanavir enhances saquinavir hard-gel concentrations in a ritonavir-boosted once-daily regimen. AIDS 2004;18:1291-1297. 
17. Boffito M, Maitland D, Dickinson L, et al.: Pharmacokinetics of saquinavir hard-gel/ritonavir and atazanavir when combined once daily in HIV Type 1-infected individuals administered different atazanavir doses. AIDS Res Hum Retroviruses 2006;22:749-756.

18. Colombo S, Buclin T, Franc C, et al.: Ritonavir-boosted atazanavir-lopinavir combination: A pharmacokinetic interaction study of total, unbound plasma and cellular exposures. Antivir Ther 2006;11:53-62.

19. Johnson M, Grinsztejn B, Rodriguez C, et al.: Atazanavir plus ritonavir or saquinavir, and lopinavir/ritonavir in patients experiencing multiple virological failures. AIDS 2005;19: 685-694.

20. Ribera E, Azuaje C, Lopez RM, et al.: Atazanavir and lopinavir/ritonavir: Pharmacokinetics, safety and efficacy of a promising double-boosted protease inhibitor regimen. AIDS 2006;20:1131-1139.

21. Ribera E, Lopez RM, Diaz M, et al.: Steady-state pharmacokinetics of a double-boosting regimen of saquinavir soft gel plus lopinavir plus minidose ritonavir in human immunodeficiency virus-infected adults. Antimicrob Agents Chemother 2004;48:4256-4262.

22. Walmsley S, Bernstein B, King M, et al.: Lopinavir-ritonavir versus nelfinavir for the initial treatment of HIV infection. N Engl J Med 2002;346:2039-2046.

23. Gilliam BL, Chan-Tack KM, Qaqish RB, Rode RA, Fantry LE, and Redfield RR. Successful treatment with atazanavir and lopinavir/ritonavir combination therapy in protease inhibitor-susceptible and protease inhibitor-resistant HIVinfected patients. AIDS Patient Care STDS 2006;20:745-759.

24. Hammer SM, Saag MS, Schechter M, et al.: Treatment for adult HIV infection: 2006 recommendations of the International AIDS Society-USA panel. Top HIV Med 2006;14:827-843.

25. Collier AC, Tierney C, Downey GF, et al.: Randomized study of dual versus single ritonavir-enhanced protease inhibitors for protease inhibitor-experienced patients with HIV. HIV Clin Trials 2008;9:91-102.

26. Gupta R, Hill A, Sawyer AW, and Pillay D. Emergence of drug resistance in HIV type 1-infected patients after receipt of first-line highly active antiretroviral therapy: A systematic review of clinical trials. Clin Infect Dis 2008;47:712-722.

27. Hosseinipour M. Resistance profile of patients failing first line ART in Malawi when using clinical and immunologic monitoring. Paper presented at: Seventeenth International AIDS Conference, 2008; Mexico City.

28. Gupta R HA, Sawyer A, Cozzi-Lepri A, et al.: The intensity of virological monitoring is associated with resistance to first line HAART in HIV-1 infected adults under the WHO public health approach to antiretroviral therapy: A systematic analysis of cohort and trial data. Lancet Infect Dis 2009;9:409-417.

29. Ledergerber B, von Overbeck J, Egger M, and Luthy R. The Swiss HIV Cohort Study: Rationale, organization and selected baseline characteristics. Soz Praventivmed 1994;39:387-394.

30. Sudre P, Rickenbach M, Taffe P, Janin P, Volkart AC, and Francioli P. Clinical epidemiology and research on HIV infection in Switzerland: The Swiss HIV Cohort Study 19882000. Schweiz Med Wochenschr 2000;130:1493-1500.

31. Grinsztejn B, Veloso VG, Pilotto JH, Campos DP, Keruly JC, and Moore RD. Comparison of clinical response to initial highly active antiretroviral therapy in the patients in clinical care in the United States and Brazil. J Acquir Immune Defic Syndr 2007;45:515-520.

32. Grinsztejn B, Nguyen BY, Katlama C, et al.: Safety and efficacy of the HIV-1 integrase inhibitor raltegravir (MK-0518) in treatment-experienced patients with multidrug-resistant virus: A phase II randomised controlled trial. Lancet 2007; 369:1261-1269.

33. Madruga JV, Cahn P, Grinsztejn B, et al.: Efficacy and safety of TMC125 (etravirine) in treatment-experienced HIV-1infected patients in DUET-1: 24-week results from a randomised, double-blind, placebo-controlled trial. Lancet 2007; 370:29-38.

34. Molina JM, Cohen C, Katlama C, et al.: Safety and efficacy of darunavir (TMC114) with low-dose ritonavir in treatmentexperienced patients: 24-week results of POWER 3. J Acquir Immune Defic Syndr 2007;46:24-31.

35. Corbett AH, Eron JJ, Fiscus SA, Rezk NL, and Kashuba AD. The pharmacokinetics, safety, and initial virologic response of a triple-protease inhibitor salvage regimen containing amprenavir, saquinavir, and ritonavir. J Acquir Immune Defic Syndr 2004;36:921-928.

36. la Porte CJ, Wasmuth JC, Schneider K, Rockstroh JK, and Burger DM. Lopinavir/ritonavir plus saquinavir in salvage therapy; pharmacokinetics, tolerability and efficacy. AIDS 2003;17:1700-1702.

37. Smith GH, Boulassel MR, Klien M, et al.: Virologic and immunologic response to a boosted double-protease inhibitorbased therapy in highly pretreated HIV-1-infected patients. HIV Clin Trials 2005;6:63-72.

38. Stebbing J, Scourfield A, Koh G, et al.: A multicentre cohort experience with double-boosted protease inhibitors. J Antimicrob Chemother 2009;64:434-435.

39. Petersen ML, Wang Y, van der Laan MJ, Rhee SY, Shafer RW, and Fessel WJ. Virologic efficacy of boosted double versus boosted single protease inhibitor therapy. AIDS 2007;21:1547-1554.

40. Loutfy M, Raboud J, Thompson C, et al.: Clinical impact of double protease inhibitor boosting with lopinavir/ritonavir and amprenavir as part of salvage antiretroviral therapy. HIV Clin Trials 2003;4:301-310.

41. Kosalaraksa P, Bunupuradah T, Engchanil C, et al.: Double boosted protease inhibitors, saquinavir, and lopinavir/ritonavir, in nucleoside pretreated children at 48 weeks. Pediatr Infect Dis J 2008;27:623-628.

42. Ananworanich J, Kosalaraksa P, Hill A, et al.: Pharmacokinetics and 24-week efficacy/safety of dual boosted saquinavir/lopinavir/ritonavir in nucleoside-pretreated children. Pediatr Infect Dis J 2005;24:874-879.

43. Ribera E and Curran A. Double-boosted protease inhibitor antiretroviral regimens: What role? Drugs 2008;68:2257-2267.

44. Glass TR, De Geest S, Hirschel B, et al.: Self-reported nonadherence to antiretroviral therapy repeatedly assessed by two questions predicts treatment failure in virologically suppressed patients. Antivir Ther 2008;13:77-85.

45. Boni J, Pyra H, Gebhardt M, et al.: High frequency of non-B subtypes in newly diagnosed HIV-1 infections in Switzerland. J Acquir Immune Defic Syndr 1999;22:174-179.

Address correspondence to: Matthias Cavassini

Infectious Diseases Service

Department of Medicine

Centre Hospitalier Universitaire Vaudois and University of Lausanne CH-1011 Lausanne Switzerland

E-mail: Matthias.Cavassini@chuv.ch 